\title{
Pharmaceutical Practice of Ceftazidime-avibactam Combined with High Dose Meropenem in XDR-Klebsiella Pneumoniae Bloodstream Infection
}

\author{
Naiju Zhang ${ }^{1}$, Jinchun Liu ${ }^{2, *}$ \\ ${ }^{1}$ Department of Pharmacy, the First Affiliated Hospital of Bengbu Medical College, Bengbu, P. R. China \\ ${ }^{2}$ Department of Pharmacy, Nanjing Drum Tower Hospital, The Affiliated Hospital of Nanjing University Medical School, Nanjing, P. R. China \\ Email address: \\ liujinchun@njglyy.com (Jinchun Liu) \\ ${ }^{*}$ Corresponding author
}

To cite this article:

Naiju Zhang, Jinchun Liu. Pharmaceutical Practice of Ceftazidime-avibactam Combined with High Dose Meropenem in XDR-Klebsiella Pneumoniae Bloodstream Infection. Journal of Drug Design and Medicinal Chemistry. Vol. 7, No. 3, 2021, pp. 50-53. doi: $10.11648 /$ j.jddmc.20210703.12

Received: September 7, 2021; Accepted: September 22, 2021; Published: October 15, 2021

\begin{abstract}
Background: The literature of ceftazidime-avibactam for bloodstream infections is limited, and whether meropenem (MIC $\geq 16 \mathrm{mg} / \mathrm{L}$ ) can be used is also a perplexing issue for clinicians. Aim: To observe the clinical efficacy and safety of ceftazidime-avibactam combined with high dose meropenem in the treatment of extensively drug-resistant (XDR) klebsiella pneumonia (Kp) bacteremia. Methods: In 1 critically ill patient with XDR-Kp diagnosed through blood culture, tigecycline combined with cefoperazone-sulbactam and tigecycline combined with polymyxin B were successively given, and both showed poor anti-infection effects. The clinical pharmacists recommend ceftazidime-avibactam combined with high dose meropenem for anti-infection therapy. Through literature review, clinical pharmacists dynamically adjusted the dose according to the creatinine clearance rate of patients. Clinical pharmacists analyzed whether meropenem (MIC $\geq 16 \mathrm{mg} / \mathrm{L}$ ) could be combined with ceftazidine-avibactam for XDR-Kp and recommended the dose of meropenem for $2 \mathrm{~g} q 8 \mathrm{~h}$ and intravenous pumping for 4 hours. Results: Ceftazidime-avibactam combined with a large dose of meropenem pumped into the vein for a long time made XDR-Kp rapidly negative conversion, with clinical symptoms, signs and inflammatory indicators such as body temperature, PCT, and CRP significantly improved, without adverse reactions such as liver and kidney function. Conclusion: Ceftazidime-avibactam combined with high dose meropenem has a significant therapeutic effect on XDR-Kp and the efficacy is safe and reliable.
\end{abstract}

Keywords: Ceftazidime-avibactam, Meropenem, XDR Klebsiella Pneumonia Bacteremia, Clinical Pharmacists, Treatment, Case Report

\section{Introduction}

In the past decade, the global prevalence of carbapenems resistant Enterobacteriaceae (CRE) infection is on the rise, especially CRE infection caused by Klebsiella pneumoniae bacteremia. According to the report of American Venture Development Company (VDC) in 2013, the incidence of Klebsiella pneumoniae bacteremia in CRE in the United States is $11 \%$, the mortality rate is nearly $50 \%$, and the treatment method is very limited. Recently, it has been reported that the resistance rate of polymyxin is worrying, especially in Klebsiella pneumoniae carbapenemases-producing K. pneumoniae (KPC-Kp) isolate. According to the data of European antibiotic resistance monitoring system in $2016,10 \%-25 \%$ of carbapenem resistant Klebsiella pneumoniae (CR-Kp) isolates in at least four southern European countries, including Italy, are resistant to polymyxin. [1-3] Avibactam, a new $\beta$-lactamase inhibitor, can effectively inhibit $\beta$-lactamases, including extended spectrum $\beta$-lactamases (ESBLs), cephalosporinase and carbapenemase. Ceftazidime combined with avibactam can significantly improve the activity of Gram-negative resistant bacteria producing $\beta$-lactamase. [4] In this paper, clinical pharmacists participated in the adjustment of the treatment plan of a case of 
extensive drug resistance Klebsiella pneumoniae (XDR-Kp), and analyzed the efficacy of ceftazidime-avibactam combined with meropenem (MIC $\geq 16 \mathrm{mg} / \mathrm{L}$ ) on XDR-Kp, so as to provide a reference for the clinical pharmacists in the treatment of CR-Kp.

\section{Case Report}

The patient, male, 62 years old, was admitted to hospital on October 16, 2018 due to multiple chest and abdomen injuries caused by falling from height. At that time, he had a clear mind. When he was sent to the local hospital, a hematoma of the right neck, collapse of the right chest wall, bone friction, positive tenderness, percussion dullness of right lower lung, coarse breath sounds at both lungs, touching pain of left upper abdomen and right upper abdomen, and limited right wrist joint were observed. Because the patient was complicated with severe hemorrhagic shock at that time, the following operations were carried out: "compression of posthepatic fissure, hemostasis with gauze packing, abdominal washing and drainage, open chest hemostasis, partial lobectomy, open reduction and internal fixation of rib fractures, and closed thoracic drainage". Due to the unstable situations after operation, the patient was admitted to intensive care unit (ICU) on October 18. Diagnosis: 1. Multiple injuries (hepatic rupture, Right pneumothorax, Neck 7 transverse process on the right, Both shoulder blades, Multiple ribs on the right side, Lumbar 4 right transverse process fracture, Left ilium, bilateral acetabulum, Pubic lower limbs, Right distal radius comminuted fracture right wrist triangular bone fracture) 2 . Acute respiratory distress syndrome (severe) 3. hypovolemic shock. 4. Acute kidney injury (KDIGO is divided into three phases) 5. Acute liver function injury. 6. After thoracoabdominal joint injury (Posterior fissure compression, Gauze packing to stop the bleeding and abdominal flushing and drainage and thoracotomy for hemostasis, Partial lobectomy, Open reduction and internal fixation of ribs and thoracic closed drainage). Because the creatinine increased to 292 umol/L, the patient was immediately treated with continuous Veno-Venous Hemofiltration $(\mathrm{CVVH})$. The patient suffered from multiple injuries-induced pulmonary hematoma, posthepatic laceration, catheterization in multiple parts of the body, continuous high fever of body temperature, showing significant increases of hemogram and $\mathrm{C}$-reactive protein (CRP). The inflammatory indices were decreased after imipenem/cilastatin sodium and linezolid anti-infection treatment, and the results of blood culture and secretion culture were negative. On October 31 , the patient suffered from sudden shiver, high fever $\left(39.9^{\circ} \mathrm{C}\right)$, blood routine examination white blood cell count $14.1 \times 10^{\wedge} 9 / \mathrm{L}$, Neutrophil percentage $88.9 \%$, procalcitonin $12.8 \mathrm{ng} / \mathrm{ml}$, C-reactive protein $122.5 \mathrm{mg} / \mathrm{L}$. The results of blood culture + drug sensitivity analysis suggested it was klebsiella pneumoniae, tigecycline-mediated, and others are drug resistance, thus the patient was diagnosed with Klebsiella pneumoniae bacteremia with multiple drug resistance. The patient was given tigecycline and cefoperazone-sulbactam (10.28-10.31), tigecycline and polymyxin B (10.31-11.7), but the anti-infectious outcomes were not effective. On November 7 , the patient was treated with ceftazidime-avibactam + meropenem. On November 11, the creatinine of the patient was decreased to $50 \mathrm{umol} / \mathrm{L}$, and CVVH was stopped. Since then, the blood culture has been negative for many times, and the inflammation indices were declined. The anti-infection course on November 26 was enough, and ceftazidime-avibactam was stopped administration. After 33 days, the results of blood culture were full-resistant $\mathrm{Kp}$, and ceftazidime-avibactam combined with meropenem was still effective.

\section{Discussion}

\subsection{Dosage Adjustment of Ceftazidime-avibactam Combined with Meropenem}

Ceftazidime-avibactam instruction pointed out in the manual that the adult loading dose of ceftazidime-avibactam was $2.5 \mathrm{~g} \mathrm{q} 8 \mathrm{~h} * 2$, infusion for $2 \mathrm{~h}$ each time, and the maintenance dose is given according to the renal function, at $2.5 \mathrm{~g} \mathrm{q} 8 \mathrm{~h}$ in case of normal renal function; when the renal function is damaged, the drug was given according to the creatinine clearance rate, namely, Clcr $31-50 \mathrm{~mL} / \mathrm{min}, 1.25$ q8h; Clcr 16-30 mL/min, 0.94 q12h; Clcr 6-15 mL/min, 0.94 q24h; Clcr $<6 \mathrm{~mL} / \mathrm{min}, 0.94 \mathrm{q} 48 \mathrm{~h}$. Patients who need dialysis are given drugs after dialysis. According to the patient's condition, it was suggested that ceftazidime-avibactam be loaded $1.25 \mathrm{q} 8 \mathrm{~h}$ and infused 2 doses every $2 \mathrm{~h}$; the maintenance dose is calculated according to the $12 \mathrm{~h}$ creatinine clearance rate, i.e. $12 \mathrm{~h}$ creatinine/blood creatinine $/ 0.72$. The creatinine clearance rate of the patient was $44 \mathrm{~mL} / \mathrm{min}$, and the clinical pharmacists suggested that ceftazidime-avibactam be infused $1.25 \mathrm{q} 8 \mathrm{~h}$ and infused 2 hours every time. Because the patient was infected by XDR-Kp blood flow, to achieve the anti-infection effect, it was recommended to use antibacterial drugs together. Because the concentration of tigecycline in blood is not high, polymyxin is easy to cause renal damage, and the patient had moderate renal damage, so clinical pharmacists recommend using meropenem. Because of patients with meropenem (MIC $\geq 16 \mathrm{mg} / \mathrm{L}$ ), clinical pharmacists recommended the use of meropenem at $2 \mathrm{~g} \mathrm{q} 8 \mathrm{~h}$, and at the same time recommended nurses to use $4 \mathrm{~h}$ of intravenous pumping. On November 13, the renal function of the patient was improved, the creatinine clearance rate was increased to $66 \mathrm{~mL} / \mathrm{min}$, and ceftazidime-avibactam was suggested at $2.5 \mathrm{~g} \mathrm{q} 8 \mathrm{~h}$.

\subsection{Efficacy of Ceftazidime-avibactam on XDR-Kp}

Ceftazidime is a well-known "third generation" $\beta$-lactamase, but avibactam represents a unique class of non $\beta$-lactamase $\beta$-lactamase inhibitors, which shows the inhibitory activities on Ambler class A, C and some d-lactamases. In vitro studies showed that ceftazidime-avibactam had a strong activity on KPC producing strains. In one study, the MIC50/90 value was $1 / 2 \mathrm{mg} / \mathrm{L}$, and in the other study, the MIC50/90 value was 
$0.5 / 2 \mathrm{mg} / \mathrm{L}$, which was far lower than the approved break point of $8 / 4 \mathrm{mg} / \mathrm{L}$. [5-8] One study reported the results of 37 patients with CRE infection treated with ceftazidime-avibactam, $59 \%$ of whom showed similar clinical cure. [9] Several studies found that ceftazidime-avibactam had a high cure rate $(74 \%)$ and a low mortality rate $(8 \%)$. [10-11] However, combination therapy accounts for $66 \%-85 \%$. In a multicenter study, the clinical results of ceftazidime-avibactam in 60 patients with CRE infection were evaluated, with $53 \%$ cure rate of pathogens and $65 \%$ clinical cure rate. Most of these patients showed highly aggressive infection of acute diseases. As mentioned earlier, about half of the patients received combined medication, the most common being aminoglycoside, polymyxin and/or tigecycline. [9] However, most of the patients (70\%) in Katie E [12] study received single drug treatment, with a clinical cure rate of $58 \%$. For patients with high risk of CRE infection caused by KPC, ceftazidime-avibactam seems to be an effective empirical treatment drug, although it is necessary to combination treatment. [9]

According to the Food and Drug Administration (FDA) approval, the indications of ceftazidime-avibactam are to treat complicated abdominal infection (combined with metronidazole), complicated urinary tract infection, hospital acquired bacterial pneumonia and ventilator-associated pneumonia. However, data from a relatively large multicenter cohort suggested that ceftazidime-avibactam may be an important option for the treatment of severe KPC-Kp infection, particularly those involving bacteremia. [13] The 30 day mortality rate of KPC-Kp bacteremia patients treated with ceftazidime-avibactam was significantly lower than that of the controls $(36.5 \%$ vs $55.7 \%, \mathrm{P}=0.005)$. The 30 day mortality rate of patients receiving ceftazidime-avibactam was significantly lower than that of patients receiving alternative single drug therapy $(9 / 22,40.9 \%$ vs $21 / 27,77.8 \%, \mathrm{P}=0.008)$. [13] In this case, when ceftazidime-avibactam combined with meropenem was used for the treatment of XDR-Kp, the blood culture turned negative in a short time. Shields et al. [14] analyzed 37 cases of CRE infection treated with ceftazidime-avibactam, and reported the recurrence rate of $5 / 37$ cases $(13.5 \%)$, while 12 cases $(8.7 \%)$ of 138 cases were reported in Tumberello $M$ et al. [13] study. In this case, the second infection occurred 33 days after ceftazidime-avibactam was stopped. The blood culture analysis was pan drug resistant (PDR) Kp. The author did not consider the recurrence of infection, because of the long term of stopping the drug, and it may be related to hospital acquired infection caused by ICU environment. In addition, 3/37 (8.1\%) cases of pneumonia reported by Shields et al were related to the resistance to ceftazidime-avibactam obtained in vitro (MICs $>8 \mu \mathrm{g} / \mathrm{mL}$ ). [14] However, in the bloodstream infection cohort of Tumbarello M' study, the event only occurred in $3 / 138(2.2 \%)$ of KPC-Kp isolates. Pneumonia is considered to be a risk factor for ceftazidime-avibactam resistance in CRE-infected patients. [15] Fortunately, the patient in this study was not resistant to ceftazidime-avibactam, and the second administration was still effective.

\subsection{Efficacy of Combination Treatment Based on Meropenem $(M I C \geq 16 \mathrm{mg} / \mathrm{L})$}

According to the principle of Enterobacteriaceae bacterial treatment, CRE is treated with the combination drug based on polymyxin or tigecycline, and can also combine other drugs based on carbapenems, such as aminoglycosides, ceftazidime-avibactam, polymyxin, tigecycline, [16] when the concentration of carbapenem is little drug resistant $(\mathrm{MIC}=4-8 \mathrm{mg} / \mathrm{L})$. Even if the MIC value reaches $16 \mathrm{mg} / \mathrm{L}$, the mortality rate of meropenem combination group is lower, and it is recommended to continue to use meropenem combination regimen when the MIC value reaches $32 \mathrm{mg} / \mathrm{L}$, and the recommended dose of meropenem is $2 \mathrm{~g} \mathrm{q} 8 \mathrm{~h}$ for $4 \mathrm{~h}$. [17] When the concentration of free carbapenems exceeds the ratio of MIC time to administration interval to $40 \%$, it has a better bactericidal effect. The $\% \mathrm{~T}>\mathrm{MIC}$ value obtained by prolonged infusion of meropenem for 4 hours can reach $75 \%$ $(\mathrm{MIC}=16 \mathrm{mg} / \mathrm{L})$. [18] It is pointed out in the specification that meropenem solution is stable within $6 \mathrm{~h}$ at $24^{\circ} \mathrm{C}$, so it is feasible to extend the infusion to 4 hours. To sum up, when the MIC of meropenem in this case is $\geq 16 \mathrm{mg} / \mathrm{L}$, the clinical pharmacists suggest that large dose of meropenem ( $2 \mathrm{~g} \mathrm{q} 8 \mathrm{~h})$ and prolonged intravenous infusion time (4h) should be used in combination with ceftazidime-avibactam to treat XDR-Kp.

\section{Conclusion}

Ceftazidime-avibactam is generally used when other anti-infection treatment programs are ineffective. Compared with other commonly used treatment programs, ceftazidime-avibactam can significantly reduce mortality, which can be used alone, but it is often used in combination, especially in combination with meropenem. Because ceftazidime-avibactam and meropenem have no serious adverse reactions related to drugs, it is of great significance to the treatment of clinical bacterial infection, especially drug-resistant bacterial infection. The limitation of this case is that the Kp gene has not been detected in both infections, and the homology analysis of $\mathrm{Kp}$ has not been done.

\section{Abbreviations}

$\mathrm{CRE}=$ carbapenems resistant enterobacteriaceae

$\mathrm{VDC}=$ venture development company

$\mathrm{Kp}=$ Klebsiella pneumonia

$\mathrm{KPC}-\mathrm{Kp}=$ Klebsiella pneumoniae

carbapenemases-producing $\mathrm{K}$. pneumoniae

$\mathrm{CR}-\mathrm{Kp}=$ carbapenem resistant Klebsiella pneumoniae

ESBLs $=$ extended spectrum $\beta$-lactamases

$\mathrm{XDR}-\mathrm{K} p=$ extensive drug resistance Klebsiella pneumoniae $\mathrm{ICU}=$ intensive care unit

$\mathrm{CVVH}=$ continuous Veno-Venous Hemofiltration

$\mathrm{CRP}=\mathrm{C}$-reactive protein

$\mathrm{PDR}=$ pan drug resistant 


\section{Author Contributions}

Writing-original draft: Naiju Zhang;

Conceptualization: Jinchun Liu;

\section{Ethic Statement}

This work was given approval by the Ethics Committee of the First Affiliated Hospital of Bengbu Medical College (2020KY072).

\section{Patient Consent}

Informed consent was obtained from the patient for publication of this case report details.

\section{Funding}

No.

\section{Conflict of Interest}

The authors of this work have nothing to disclose.

\section{Acknowledgements}

The authors are grateful to a patient who gave his informed consent for publication.

\section{References}

[1] Giacobbe DR, Del Bono V, Trecarichi EM, et al; ISGRI-SITA (Italian Study Group on Resistant Infections of the Società Italiana Terapia Antinfettiva). Risk factors for bloodstream infections due to colistin-resistant KPC-producing Klebsiella pneumoniae: results from a multicenter case-controlcontrol study. Clin Microbiol Infect, 2015; 21: 1106. e1-8.

[2] European Centre for Disease Prevention and Control (ECDC). Summary of the latest data on antibiotic resistance in EU: 2016. Available

https://ecdc.europa.eu/en/publicationsdata/summary-latest-dat a-antibiotic-resistance-eu-2016. Accessed 19 March 2018.

[3] Rojas LJ, Salim M, Cober E, et al; Antibacterial Resistance Leadership Group. Colistin Resistance in Carbapenem-Resistant Klebsiella pneumoniae: Laboratory Detection and Impact on Mortality. Clin Infect Dis, 2017; 64: 711-718.

[4] Lin Huizhen, Fang Songbai, Zhao Hongwei, et al. Ceftazidime-avibactam, a new antibacterial agent for Gram-negative bacterial infection [J]. Herald of Medicine, 2016, 35 (7): 735-739.

[5] Shields RK, Clancy CJ, Hao B et al. Effects of Klebsiella pneumoniae carbapenemase subtypes, extended-spectrum b-lactamases, and porin mutations on the in vitro activity of ceftazidime-avibactam against carbapenemresistant K. pneumoniae. Antimicrob Agents Chemother 2015; 59: 5793-7.
[6] Castanheira M, Farrell SE, Krause et al. Contemporary diversity of b-lactamases among Enterobacteriaceae in the nine U.S. census regions and ceftazidime-avibactam activity tested against isolates producing the most prevalent b-lactamase groups. Antimicrob Agents Chemother 2014; 58: 833-8.

[7] Clinical and Laboratory Standards Institute. Performance Standards for Antimicrobial Susceptibility Testing: Twenty-Second Informational Supplement M100-ED28. CLSI, Wayne, PA, USA, 2018.

[8] EUCAST. Breakpoint Tables for Interpretation of MICs and Zone Diameters, Version 8.0, 2018. http://www.eucast.org.

[9] King M, Heil E, Kuriakose S et al. Multicenter study of outcomes with ceftazidime-avibactam in patients with carbapenem-resistant Enterobacteriaceae infections. Antimicrob Agents Chemother 2017; 61: e00449-17.

[10] Temkin E, Torre-Cisneros J, Beovic B et al. Ceftazidime-avibactam as salvage therapy for infections caused by carbapenem-resistant organisms.

[11] Antimicrob Agents Chemother 2017; 61: e01964-16.36 van Duin D, Lok JJ, Earley $M$ et al. Colistin versus ceftazidime/avibactam in the treatment of infections due to carbapenem-resistant Enterobacteriaceae. Clin Infect Dis 2017; 66: $163-71$.

[12] Katie E. Barber, Jason M. Pogue, Henderson D. et al. Ceftazidime/avibactam versus standard-of-care agents against carbapenem-resistant Enterobacteriaceae harbouring blaKPC in a one-compartment pharmacokinetic/pharmacodynamic model [J]. J Antimicrob Chemother. 2018 Sep 1; 73 (9): 2405-2410.

[13] Tumbarello M, Trecarichi EM, Corona A, et al. Efficacy of Ceftazidime-Avibactam Salvage Therapy in Patients With Infections Caused by Klebsiella pneumoniae Carbapenemase-producing K. pneumoniae. Clin Infect Dis. 2019 Jan 18; 68 (3): 355-364.

[14] Shields RK, Potoski BA, Haidar G, et al. Clinical Outcomes, Drug Toxicity, and Emergence of Ceftazidime-Avibactam Resistance Among Patients Treated for Carbapenem-Resistant Enterobacteriaceae Infections. Clin Infect Dis, 2016; 63: 1615-1618.

[15] Shields RK, Nguyen MH, Chen L, et al. Pneumonia and Renal Replacement Therapy Are Risk Factors for Ceftazidime-Avibactam Treatment Failures and Resistance among Patients with Carbapenem-Resistant Enterobacteriaceae Infections [J]. Antimicrob Agents Chemother. 2018 Apr 26; 62 (5). pii: e02497-17.

[16] Expert consensus on clinical application of pharmacokinetic/pharmacodynamic theory of antibacterial drugs, Chin J Tuberc Respir Dis, 2018, 41 (6): 409-446.

[17] Petrosillo N, Giannella M, Lewis R, et al. Treatment of carbapenem-resistant Klebsiella pneumoniae: The state of the art [J]. Expert Rev Anti Infect Ther, 2013, 11 (2): 159-177.

[18] Daikos G L, Markogiannakis A. Carbapenemase-producing Klebsiella pneumoniae: (When) might we still consider treating with carbapenems [J]. Clin Microbiol Infect, 2011, 17 (8): 1135-1141. 